\title{
Adenomiosis de vesícular biliar
}

Gallbladder adenomyosis

Gabriela Espinosa ${ }^{1}$ Luis Ruso Martinez ${ }^{2}$.

DOI: $10.31837 /$ cir.urug/2.1.5

Recibido: 25 de enero de 2018

Aceptado: 23 de abril de 2018

La adenomiomatosis vesicular (AV)se observa entre 1\%y8,5 \% de las colecistectomías(1,2). Se asocia a colelitiasis en $80 \%$ de los casos.(3)

Es una neoplasia benigna, caracterizada por hiperplasia del epitelio vesicular con invaginaciones hasta la subserosao serosa formando divertículos intramurales, los senos de Rokitansky-Aschoff.(4) (Figura 1)
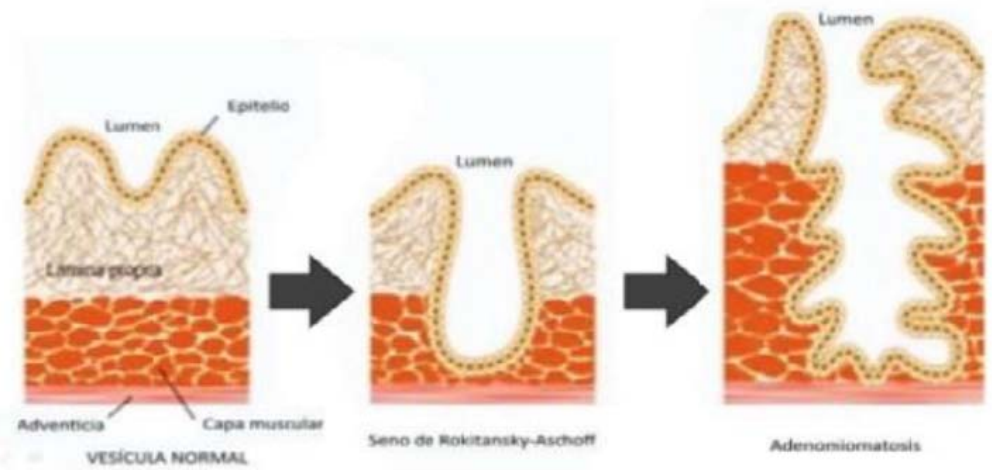

Fig. 1 Formación de los senos de Rokitansky -Aschoff.

Se observa la hipertrofia muscular asociada a invaginación de la mucosa de diversa profundidad. La ecografía es relativamente inespecífica; pero la TC contrastada incrementa el diagnóstico .

La colangioRNM es studio más eficaz con una sensibilidad de $81 \%$ a $93 \%$ y especificidad de $93 \%$ a $100 \%$; porque las cavidades intraluminares hiperintensas en T.2 son sugestivas de ser senos de Rokitansky-Asffof y excluyen el diagnóstico de cáncer de vesícula.(2,5,6). Aún así, el 3.2 \% de las $\mathrm{AV}$, se asocian con cáncer precoz de vesícula biliar (3) y el tipo segmentario del fondo vesicular es considerada una condición preneoplasica.(3)

${ }^{1}$ Clinica Quirúrgica“3” Hospital Maciel. Facultad de Medicina. Universidad de la República Montevideo, Uruguay gabrielaespinosa@msn.com

${ }^{2}$ Prof. Clínica Quirúrgica 3, Hospital Maciel. Facultad de Medicina. Universidad de la República Montevideo, Uruguay 
Se presentan una paciente de 56 años, colecistectomizada por litiasis y adenomiomatosis de vesícula biliar. (Figuras 2 y 3 )

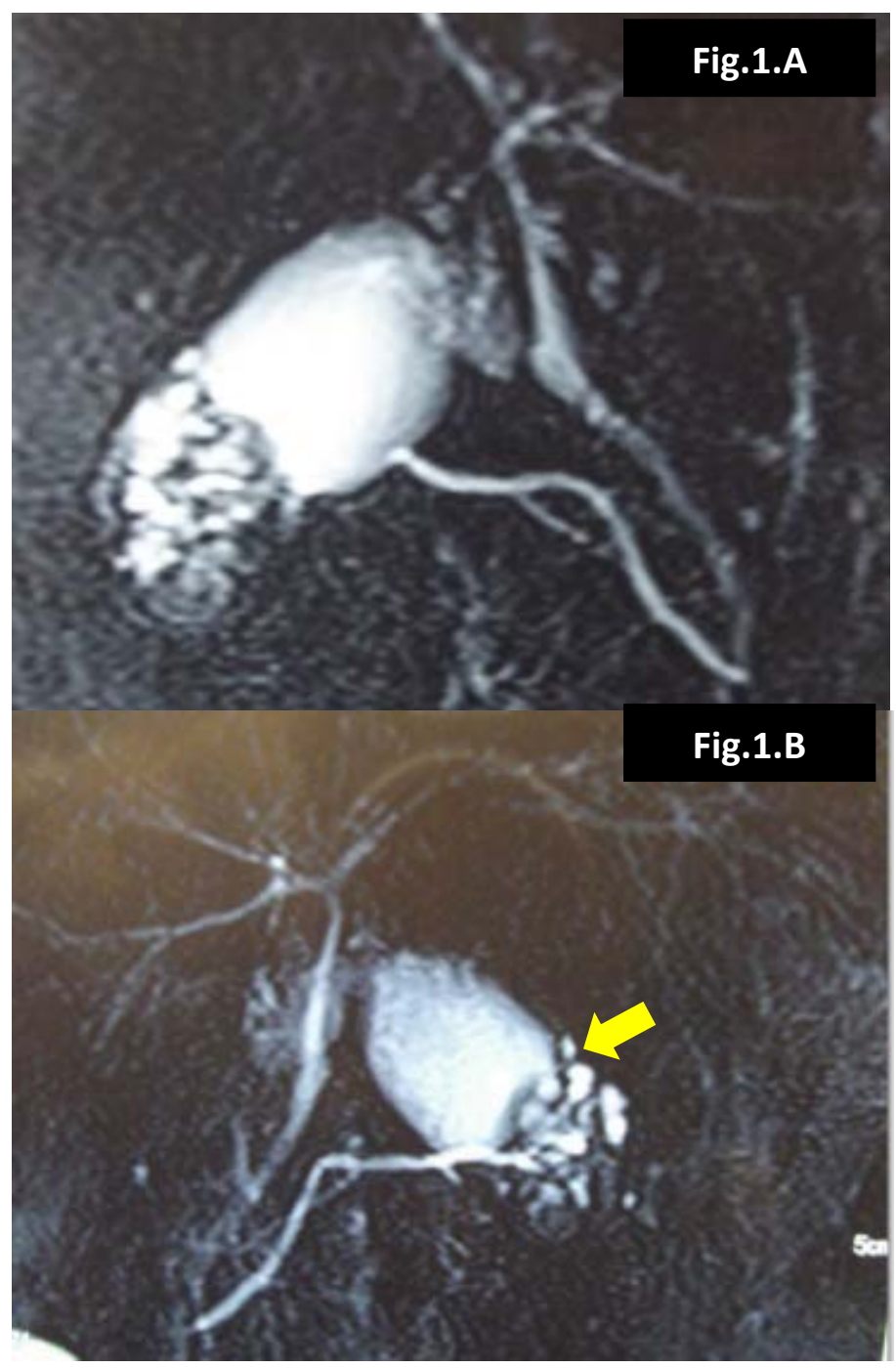

Fig. 2 CRNM. Vista anterior (1.A) y posterior (Fig. 1.B) de una adenomiomatosis segmentaria. Se observa engrosamiento parietal con focos hipercaptantes en el fondo. En T2 se puede visualizar los senos de RokitanskyAschoff (flecha) que permiten diferenciar la hiperplasia adenomiomatosa del carcinoma vesicular 


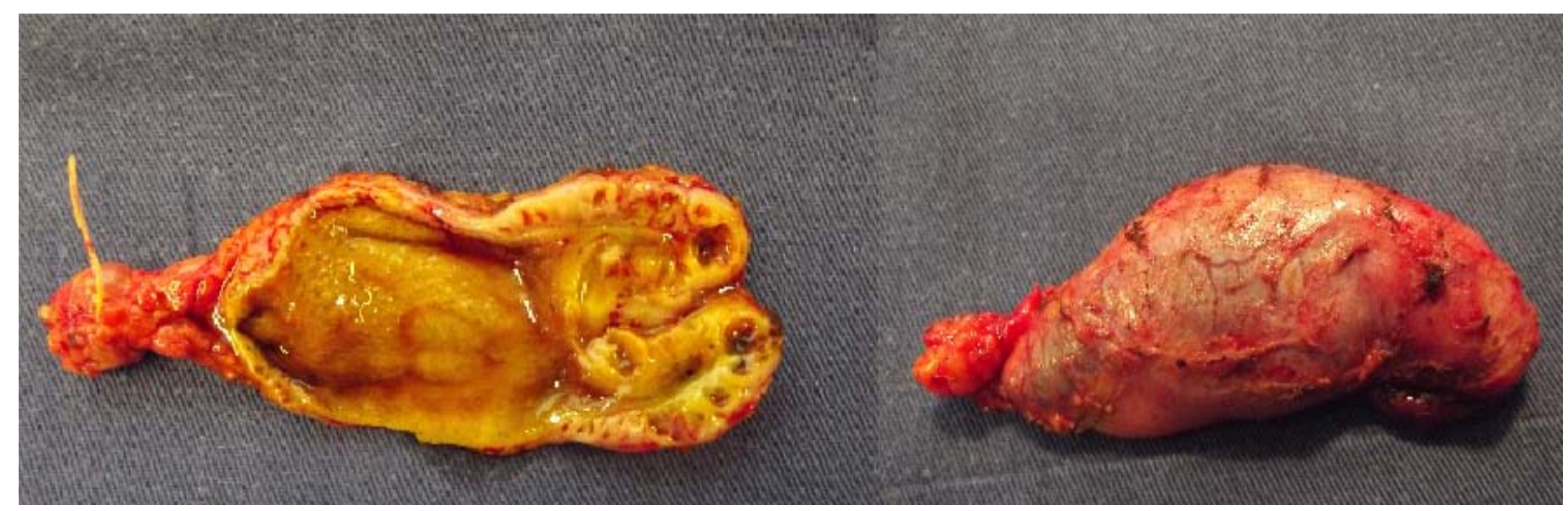

Fig. 3 PATOLOGIA. Imagen de la vesícula cerrada (der) abierta y evacuada (izq.) .En el fondo área de 20x20x20 mm con cavidad de aproximadamente $4 \mathrm{~mm}$ con tractos gruesos interpuestos. En sector proximal espesor parietal de $5 \mathrm{~mm}$ con mucosa reticular (Flecha) .

\section{Bibliografía}

1- Bang, S., Lee J., Woo H., Joo I., Lee E., Han J., Choi B. Differentiating between adenomyomatosis and gallbladder cancer: revision a comparative study of High-resolution ultrasound,multidetector CT and MR imaging. Korean J. REadiol 2014;15:226-34

2- Mahajan A., Sripathi S.Gallbladder adenomyomatosis mimicking carcinoma: a diagnostic dilemma.JGO 2016;2:341-5

3- Morikawa T., Okabayashi T., Shima Y., Sumiyoshi T., Kokuzi A., Saika Y., et al. Adenomyomatosisconcomitantwithprimarygallbladder carcinoma. Acta Med. Okayama 2017; 71:113-8.

4. Jutras JA: Hyperplastic cholecystoses; Hickey lecture, 1960. Am J Roentgenol Radium TherNucl Med 1960, 83 :795-827

5- Bonatti M., Vezzali N., Lombardo F., Ferro F., Zamboni G., TauberM.,et al. Gallbladder adenomyomatosis: imaging findings ,tricks and pitfalls. Insights Imaging 2017; 8:24353.

6- Hammad A., Miura J., Turaga K., Johnston F., Hohenwalter M., Gambin C. A literature review of radiological findings to guide the diagnosis of gallbladder adenomyomatosis. HPB 2016; 18: 129-35. 